\title{
Magnetic resonance vessel wall imaging in cerebrovascular diseases
}

\author{
Christopher C. Young, MD, PhD, ${ }^{1}$ Robert H. Bonow, MD, ${ }^{1}$ Guilherme Barros, MD, ${ }^{1}$ \\ Mahmud Mossa-Basha, MD, ${ }^{2}$ Louis J. Kim, MD, ${ }^{1,2,4}$ and Michael R. Levitt, MD ${ }^{1-4}$
}

Departments of ${ }^{1}$ Neurological Surgery, ${ }^{2}$ Radiology, and ${ }^{3}$ Mechanical Engineering, and ${ }^{4}$ Stroke and Applied Neuroscience Center, University of Washington, Seattle, Washington

Cerebrovascular diseases manifest as abnormalities of and disruption to the intracranial vasculature and its capacity to carry blood to the brain. However, the pathogenesis of many cerebrovascular diseases begins in the vessel wall. Traditional luminal and perfusion imaging techniques do not provide adequate information regarding the differentiation, onset, or progression of disease. Intracranial high-resolution MR vessel wall imaging (VWI) has emerged as an invaluable technique for understanding and evaluating cerebrovascular diseases. The location and pattern of contrast enhancement in intracranial VWI provides new insight into the inflammatory etiology of cerebrovascular diseases and has potential to permit earlier diagnosis and treatment. In this report, technical considerations of VWI are discussed and current applications of VWI in vascular malformations, blunt cerebrovascular injury/dissection, and steno-occlusive cerebrovascular vasculopathies are reviewed.

https://thejns.org/doi/abs/10.3171/2019.9.FOCUS19599

KEYWORDS intracranial vessel wall imaging; cerebral aneurysm; endothelial inflammation; vasospasm; intracranial atherosclerosis; moyamoya

$\mathrm{C}$ EREBROVASCULAR diseases manifest as abnormalities and disruption to the intracranial vasculature and its capacity to deliver oxygenated blood to the brain. ${ }^{37}$ Luminal imaging such as CT angiography (CTA), MR angiography (MRA), and digital subtraction angiography (DSA), and perfusion techniques such as CT perfusion and PET have been of tremendous value in the diagnosis and management of cerebrovascular disease. However, evaluation of luminal anatomy and perfusion may not be adequate, because cerebrovascular pathology often begins in the vessel walls. ${ }^{29,37}$ Intracranial vessel wall imaging (VWI) has emerged as an invaluable technique to characterize inflammatory changes in the vessel wall. ${ }^{28}$ Vessel wall changes frequently precede anatomical and functional perfusion changes. ${ }^{28,29,37}$ Therefore, VWI may be useful in the prediction and early detection of cerebrovascular diseases.

The first intracranial VWI study was performed in
1995, when investigators reported age-related changes in the degree of carotid artery (CA) and vertebral artery (VA) contrast enhancement. ${ }^{2}$ VWI has since been applied to the evaluation of cerebrovascular conditions including cerebral aneurysms, post-subarachnoid hemorrhage (SAH) vasospasm, traumatic blunt cerebrovascular injury (BCVI), and other steno-occlusive conditions. ${ }^{29,37}$

\section{Technical Aspects}

To accurately characterize lesions within thin vessel walls, a high contrast-to-noise ratio (CNR) and spatial resolution are critical. The CNR is the signal contrast between the vessel wall and the surrounding luminal blood and CSF. Suppression of luminal blood depends on intrinsic "black blood" quality of 2D and 3D acquisition sequences. The vessel wall should be visualized in multiple axes. Using multiple orthogonal 2D sequences is one

ABBREVIATIONS $A C O A=$ anterior communicating artery; $A V M=$ arteriovenous malformation; $B C V I=$ blunt cerebrovascular injury; $C A=$ carotid artery; $C N R=$ contrast-tonoise ratio; $C T A=C T$ angiography; DANTE = delayed alternating nutation for tailored excitation; DSA = digital subtraction angiography; ICA = internal carotid artery; MCA = middle cerebral artery; MMD = moyamoya disease; $\mathrm{MMS}$ = moyamoya syndrome; $\mathrm{MRA}=\mathrm{MR}$ angiography; $\mathrm{MSDE}=$ motion-sensitized driven equilibrium; $\mathrm{PC} \mathrm{A}=$ posterior communicating artery; PHASES = population, hypertension, age, size of aneurysm, earlier SAH from another aneurysm, and site of aneurysm; RCVS = reversible cerebral vasoconstriction syndrome; $\mathrm{SAH}=$ subarachnoid hemorrhage; SPACE = sampling perfection with application-optimized contrasts by using different flip angle evolutions; VA = vertebral artery; VISTA = volume isotropic turbo spin echo acquisition; VWCE = vessel wall contrast enhancement; VWI = vessel wall imaging.

SUBMITTED July 31, 2019. ACCEPTED September 4, 2019.

INCLUDE WHEN CITING DOI: 10.3171/2019.9.FOCUS19599. 


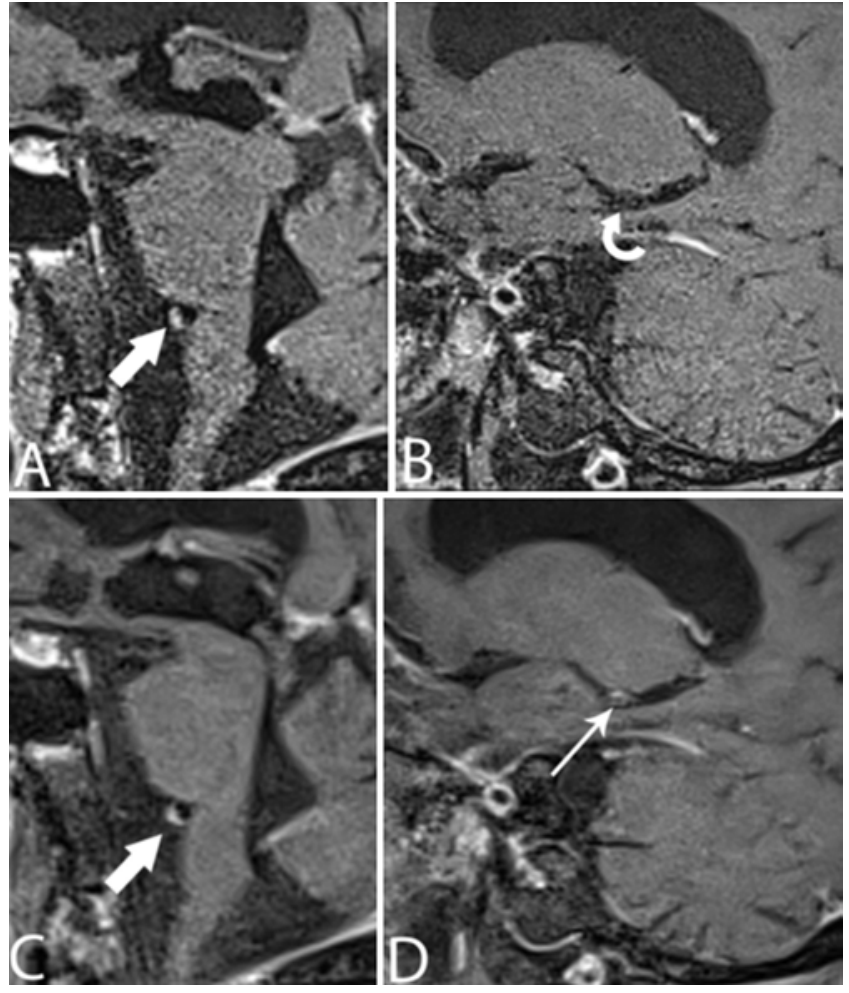

FIG. 1. Optimized blood and CSF suppression to reduce artifacts in VWI. Suppression of artifacts is important in VWI. DANTE is a preparation pulse that suppresses CSF and blood flow signal (A and B). Eccentric vessel wall enhancement is seen in both DANTE (A, bold arrow) and non-DANTE (C, bold arrow) sagittal T1 SPACE VWI, representing intracranial atherosclerosis. However, DANTE suppresses nonspecific enhancement in a vein (B, curved arrow), which enhances on the nonDANTE sequence (D, arrow).

method, but partial volume averaging can obscure the arterial wall's appearance. With 2D VWI, double inversion recovery pulses can be used to generate blood flow suppression. Spin-echo imaging techniques inherently also generate dark luminal blood, but are limited by slow or in-plane flow resulting in loss of suppression..$^{29}$

Newer 3D VWI volumetric acquisitions enable increased brain coverage and through-plane resolution, with isotropic scans reformatted in multiple planes. These include variable refocusing flip angle sequences (VRFA), with $\mathrm{T} 1$ or proton density-weighted pre- and postcontrast sequences, such as volume isotropic turbo spin echo acquisition (VISTA; Philips Healthcare), sampling perfection with application-optimized contrasts by using different flip angle evolutions (SPACE; Siemens), and Cube software (GE Healthcare). Preparation pulses, such as motion-sensitized driven equilibrium (MSDE), which uses flow-sensitive dephasing gradients, also achieve blood suppression. Another approach to optimize blood and CSF suppression is delayed alternating nutation for tailored excitation (DANTE), a preparation pulse that uses a series of low flip angle nonselective pulses interleaved with gradient pulses of short repetition times. . $^{18,32,37,63}$

Laminar blood flow through pathological segments is often slow, turbulent, or stagnant. Vessel wall contrast en- hancement occurs at sites of pathological instability and inflammation. However, because gadolinium shortens T1 relaxation time, "black blood" suppression in areas of abnormal or even normal in-plane flow can be diminished. This potentially causes an artifact of unsuppressed contrast in flowing blood, appearing as vessel wall contrast enhancement (VWCE). This is seen with turbulence and recirculation within aneurysm sacs, slow flow within dilated lumens, and retrograde filling of distal collateral branches with proximal occlusion. ${ }^{32}$ This can also be seen in certain arterial segments in healthy patients on postcontrast 3D VWI, specifically with in-plane arteries. DANTE preparation pulses suppressed near-wall enhancement by $50 \%$ compared to 3D turbo spin echo alone, underscoring the significance of artifact and the value of preparation pulses (Fig. 1). ${ }^{26}$

Other sources of misinterpreted VWCE include vasa vasorum, adjacent veins, and endovascular interventions such as mechanical thrombectomy. ${ }^{32}$ With age-dependent atherosclerosis, the extracranial vasa vasorum network can extend into the proximal intracranial arteries to supply stenotic vessels. ${ }^{2}$ This phenomenon can also happen distally, with smaller intracranial branches supplying the adventitia of larger, stenotic vessels. This can appear as concentric (circumferential) VWCE and can mimic inflammatory vasculitis. The differentiating feature between vasa vasorum and inflammatory vasculopathy is the lack of arterial stenosis and wall edema with vasa vasorum ingrowth. Veins traveling adjacent to the artery of interest may appear as VWCE on 2D VWI, but this is not typically a problem with 3D VWI. Imaging in a second orthogonal plane can clarify this. Recanalization of occluded arteries after mechanical thrombectomy causes concentric VWCE, similar in appearance to inflammatory vasculitis, probably secondary to arterial wall injury, and has associations with longer procedures with multiple passes and subsequent hemorrhagic conversion. ${ }^{32,56}$

Scanning at higher field strengths in which 3D VWI techniques are used provides improved through-plane resolution, signal-to-noise ratio, imaging reproducibility, and CNR. CSF flow can result in artifacts that can mimic arterial wall abnormalities. Incorporating an antidriven equilibrium pulse at the end of the echo train to a 3D turbo spin echo acquisition can reduce tissue signals with long T2 relaxation times, such as CSF, independent of flow. Augmentation with antidriven equilibrium provides $50 \%$ improvement in the $\mathrm{M}_{1}$ segment and $183 \%$ improvement in the basilar artery for wall-to-CSF CNR. ${ }^{72}$ DANTE preparatory pulses can also suppress CSF signal and flow artifacts when used with 3D VWI techniques. ${ }^{68}$

Intracranial vessel walls are challenging to image due to their tortuosity and small caliber. The thickness of the proximal middle cerebral artery (MCA) wall varies from $0.4 \mathrm{~mm}$ to $0.7 \mathrm{~mm}$, with a mean outer diameter of 3.4 mm. ${ }^{1,46}$ A recent ex vivo study of VWI of the circle of Willis showed accurate reliability comparing histological and 7-T MR measurements across entire arterial segments. ${ }^{22}$ Currently, a resolution of $3 \mathrm{~T}$ (0.4- to $0.5-\mathrm{mm}$ isotropic voxel size) is preferred in the clinical setting-balancing cost, acquisition time, image quality, diagnostic accuracy, and clinical need. ${ }^{29,37}$ 

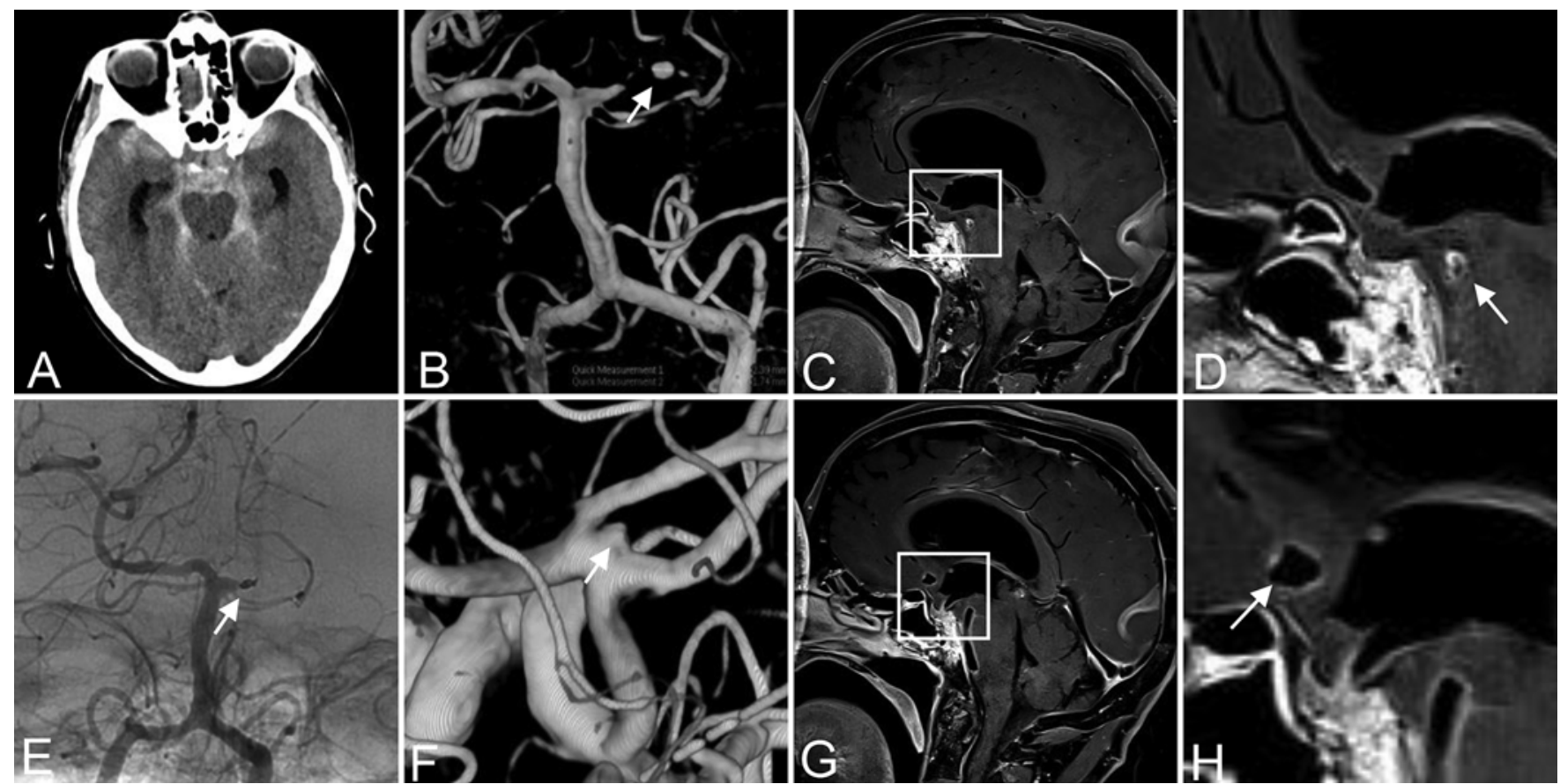

FIG. 2. Contrast enhancement in VWI identifies the ruptured aneurysm. A patient presented with high-grade SAH (A) and multiple aneurysms on angiogram; a left posterior cerebral artery (PCA) dissecting aneurysm (B, arrow) and an ACoA aneurysm ( $\mathbf{F}$, arrow). VWI demonstrates significant wall enhancement involving the PCA aneurysm (C) suggesting this as the source of SAH. The white boxed area is enlarged in D; arrow designates the PCA aneurysm. The unruptured ACoA aneurysm does not enhance more than the surrounding arterial walls $(\mathbf{G})$. The white boxed area is enlarged in $\mathbf{H}$; arrow designates the ACoA aneurysm. The patient underwent successful endovascular coiling of the ruptured left PCA aneurysm (E, arrow).

In 2018, the American Society of Neuroradiology formulated guidelines for the applications of VWI..$^{32}$ The current consensus advocates for pulse sequences sufficient for blood and CSF suppression, 2D sequences in short- and long-axis planes, and 3D sequences with isotropic voxel dimensions and multiplanar reformatting.

\section{Current Applications of VWI Vascular Malformations \\ Cerebral Aneurysms}

Cerebral aneurysms are the manifestation of different pathological processes involving the intracranial cerebral vessel wall. ${ }^{27,28}$ Traditional imaging, including DSA, informs luminal caliber and alterations to intracranial blood flow. For evaluation of saccular aneurysms, this provides anatomical information including size and location, forming the basis for risk quantification systems such as the population, hypertension, age, size of aneurysm, earlier SAH from another aneurysm, and site of aneurysm (PHASES) classification system. ${ }^{20}$ However, aneurysm size-based scoring systems are imperfect because the majority of ruptured aneurysms are small., 54

VWCE reflects the degree of vessel wall inflammation, which is characterized by vessel wall thickening, development of vasa vasorum, and macrophage infiltration. ${ }^{57}$ VWI provides information regarding 1) stability of the aneurysm wall and risk of aneurysm rupture, and 2) discrimination between ruptured and unruptured aneurysms (in a patient with multiple aneurysms). VWI of aneurysm walls has demonstrated increased enhancement in growing and recently ruptured aneurysms..$^{23,30,45,67}$ VWCE has been correlated with inflammatory changes at a histological level, ${ }^{57}$ and has been associated with inflammatory vasculopathies such as mycotic aneurysms and HIV-associated vasculitis. ${ }^{28}$

Multiple Cerebral Aneurysms. When a patient with multiple intracranial aneurysms presents with acute SAH, the offending aneurysm may not be easy to identify. ${ }^{51}$ However, aneurysm rupture is associated with VWCE with high sensitivity and can be used to identify the ruptured aneurysm, facilitating surgical decision-making (Fig. 2). After the initial report by Matouk et al., ${ }^{34}$ subsequent studies have examined more than 500 aneurysms and found that $>95 \%$ of ruptured aneurysms demonstrated enhancement. $17,25,42,45,64,67$ Approximately $25 \%$ of unruptured aneurysms also demonstrated enhancement; many of them were deemed unstable based on documented growth or symptoms. These results suggest that VWI has a high sensitivity and negative predictive value for identifying acute ruptured aneurysms. ${ }^{51,64,73}$

Stability of Cerebral Aneurysms. Unstable aneurysms are at higher risk of rupture and warrant treatment. Instability is based on aneurysm size, morphology, documented growth, and/or clinical symptoms. In addition to established risk-quantification strategies such as the PHASES score ${ }^{20} \mathrm{VWI}$ provides an additional method to evaluate aneurysm stability. ${ }^{51}$ VWCE has been associated 

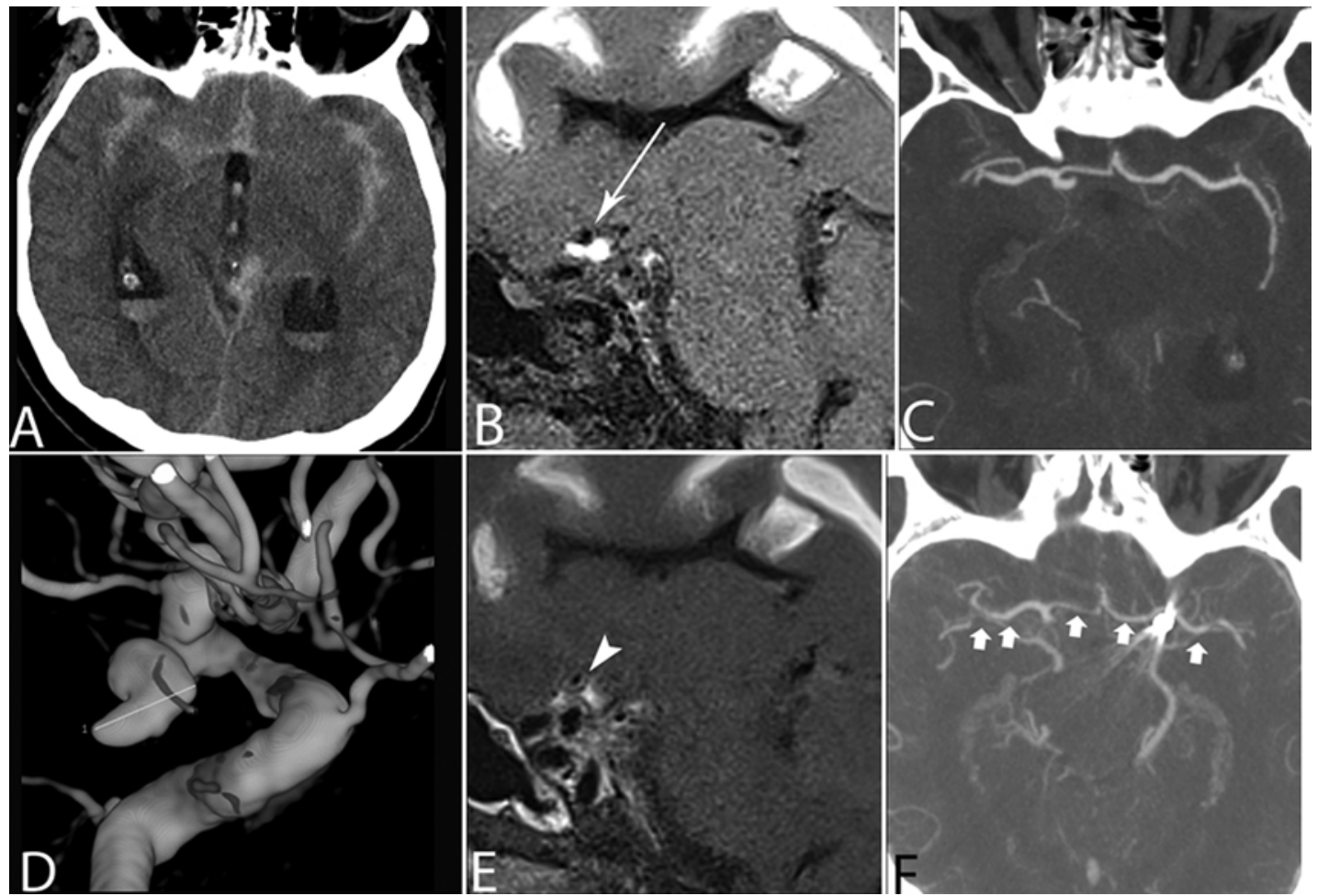

FIG. 3. Vasospasm on VWI. The patient presented with SAH secondary to a ruptured left PCoA aneurysm (A and D). VWI obtained 24 hours after admission demonstrates left MCA (B, arrow, precontrast) with circumferential vessel wall enhancement (E, arrowhead, postcontrast). In comparison to the initial CTA, which shows normal-caliber vessels $(\mathbf{C})$, there is diffuse bilateral vasospasm 8 days postictus ( $\mathbf{F}$, arrows).

with high-risk features and instability in unruptured aneurysms. ${ }^{23,30,31,66}$ In 140 unruptured aneurysms, VWCE was associated with greater mean aneurysm size (10.4 vs 5.6 $\mathrm{mm}$ ) and a higher-risk location (anterior communicating artery $[\mathrm{ACoA}]$, posterior communicating artery $[\mathrm{PCoA}]$, and posterior circulation). In one study, $92.3 \%$ of aneurysms with a PHASES score $>10$ were enhanced compared with $33.9 \%$ of aneurysms scoring $<4 .{ }^{30}$ Similarly, dichotomizing 45 patients into PHASES scores of either $\geq 3$ or $<3$, our group reported VWCE in $42.1 \%$ versus $14.8 \%$, respectively. ${ }^{23}$

There are limitations to this application. VWI and VWCE patterns may be misleading, with both false positives and false negatives for evaluating aneurysm rupture. In one reported case, a patient with acute $\mathrm{SAH}$ was found to have 2 lesions: a 3.5-mm MCA aneurysm and a $1.5-\mathrm{mm}$ PCoA aneurysm. ${ }^{73}$ On VWI, the MCA aneurysm demonstrated circumferential enhancement, whereas the PCoA aneurysm did not enhance. Intraoperatively, the PCoA aneurysm was found to be acutely ruptured, in contradiction to the VWI result.

The significance of VWCE in unruptured aneurysms without obvious unstable features remains unclear. This is a clinically important group, because these aneurysms are often observed. Future studies will need to examine whether VWCE in this so-called low-risk group is predictive of instability and higher risk of rupture, thereby warranting prompt treatment.

Vasospasm. VWI may predict and detect vasospasm following SAH and aneurysm treatment. In a cohort of unruptured and ruptured aneurysms ${ }^{39}$ in which VWI was obtained soon after endovascular treatment (prior to the classic onset of vasospasm and delayed cerebral ischemia), early VWCE in second- and third-order intracranial arteries was significantly associated with development of angiographic vasospasm, even after controlling for modified Fisher grade (Fig. 3). Intracranial arterial segments were also more likely to show enhancement following aneurysm rupture and modified Fisher grade $4 \mathrm{SAH}$. It is possible that VWCE reflects inflammatory changes that are more robust following aneurysm rupture and highgrade aneurysmal $\mathrm{SAH}$, correlating with the risk of developing vasospasm. Although the extent of SAH is the most reliable predictor of vasospasm, the clinical severity of vasospasm varies considerably and can occur even with low-grade SAH. VWI may have a valuable role in the prediction of vasospasm in otherwise low-risk patients.

Follow-Up of Flow-Diverter-Treated Aneurysms. VWCE is frequently seen following balloon- or stent-assisted endovascular treatment or deployment of flow diverters. Although the mechanism of VWCE is unclear, it probably represents mechanical irritation that resolves over time, ${ }^{28,35,39}$ and is thought to be related to an inflammatory process in the vessel wall and the transient breakdown of the blood-brain barrier. Posttreatment VWCE does not appear to be associated with instability and increased risk of aneurysm rupture. ${ }^{35}$ Further study is needed to determine whether VWCE, either at the time of treatment or at long- 

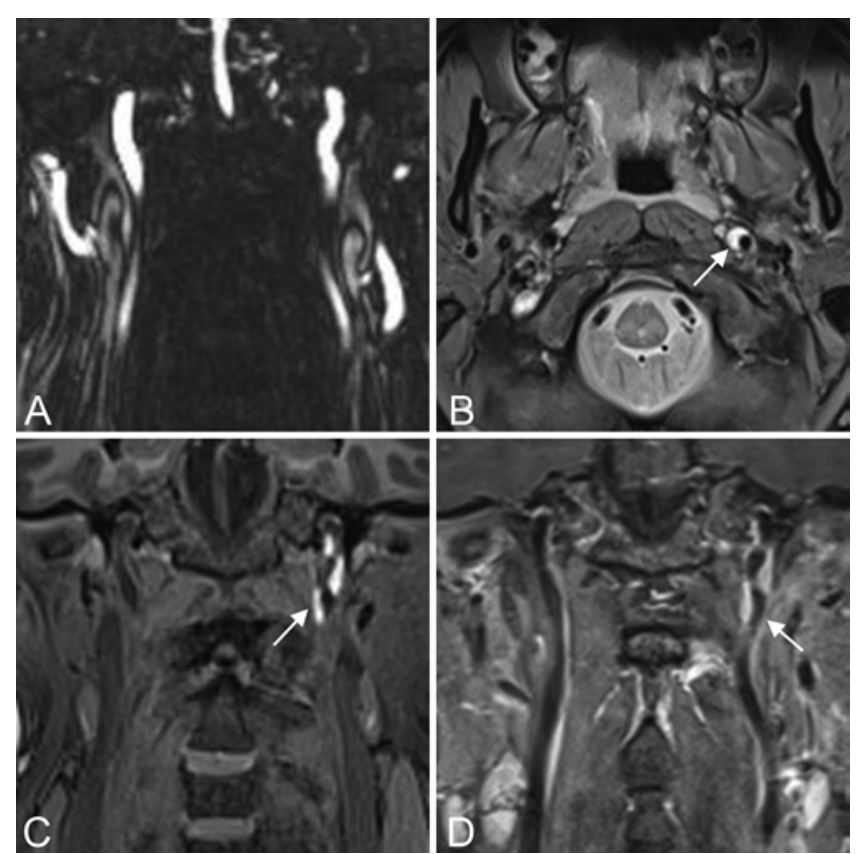

FIG. 4. VWI in a CA dissection. CA dissection may be difficult to visualize on luminal imaging such as MRA $(\mathbf{A})$ if stenosis is not present, specifically in a subadventitial dissection. On axial proton density-weighted VWI (B) crescentic hyperintensity can be seen, which represents intramural hematoma (arrow). Coronal T1-weighted pre- and postcontrast VWI (C and D, respectively) demonstrates intramural thrombus (C, arrow) with associated vessel wall enhancement surrounding the hematoma (D, arrow).

term follow-up, correlates with treatment outcome and aneurysm obliteration.

\section{Arteriovenous Malformations}

The results from A Randomized Trial of Unruptured Brain Arteriovenous Malformations (ARUBA) support the nonsurgical treatment of unruptured high-grade arteriovenous malformations (AVMs). ${ }^{36}$ However, when an AVM presents with acute hemorrhage, it is reasonable to identify high-risk features, such as intranidal and flow-related aneurysms that may be the source of the hemorrhage, and to target these lesions for intervention. ${ }^{33}$ Identification and treatment of the rupture point in a high-grade complex AVM is often difficult. In several reports, VWI has demonstrated promise in identifying the rupture point by the presence of significant VWCE. ${ }^{7,44}$ Additional evidence is required prior to widespread clinical adoption, however.

\section{Dissection and BCVI}

\section{CA and VA Dissection}

Spontaneous arterial dissections are an important cause of stroke especially in young patients,,${ }^{52,58}$ and VWI may facilitate identification and characterization of these lesions. CA and VA dissections occur intracranially and extracranially. ${ }^{52,58}$ Dissections occur when a tear in the intimal layer allows blood under arterial pressure to elevate a subintimal and/or subadventitial flap, creating a false lumen and an associated intramural hematoma. ${ }^{52}$ Dissections and the associated hematoma can cause stroke by stenosis of the artery, occlusion of the branching vessels' ostia, or formation of arterial thromboemboli. ${ }^{52}$ Subintimal dissections communicate with the lumen and are often associated with stenosis of the artery and thromboembolism. Subadventitial dissections may lead to pseudoaneurysm formation, with risk of rupture and SAH. Due to the absence of external elastic lamina and a thinner adventitial layer in the intradural vasculature, intracranial dissections tend to occur in the subadventitial plane. Angiography may incompletely image these lesions, particularly if there is minimal arterial stenosis or the false lumen is thrombosed (Fig. 4). ${ }^{58}$

High-resolution VWI may help delineate important anatomical details or reveal lesions not evident with other imaging modalities. ${ }^{11}$ Typical findings include a dissection flap, an intramural thrombus, and abnormal enhancement. Dissection flaps may not be detected reliably, ${ }^{43}$ but intramural hematomas are seen in $87 \%-100 \%$ of cases. ${ }^{10,29,43,61,62}$ Detection is aided by the inclusion of susceptibility-weighted and noncontrast T1-weighted sequences. ${ }^{10,29}$ The majority are hyperintense on T1- and T2weighted sequences (74\% and $60 \%$, respectively), with the remainder demonstrating iso- or hypointense signal intensity on T1- and T2-weighted sequences. As with intracerebral hematomas, the signal characteristics of intramural hematomas change predictably with time. Thus, MRI appearance can define the chronicity of the injury. ${ }^{21}$

VWCE is evident in approximately two-thirds of dissections. ${ }^{3,14,47,61}$ When present, this finding is thought to be due to inflammation, stasis of blood within the false lumen, or disruption of the vasa vasorum. ${ }^{48,50}$ The enhancement pattern tends to be eccentric $(<50 \%$ circumference $),{ }^{48,61}$ and may extend beyond the visualized dissection flap and/ or hematoma, suggesting farther-reaching injury to the intima than can be seen on noncontrasted sequences (Fig. 4). ${ }^{3}$ Extensive enhancement in cervical dissections may be associated with a heightened risk of multiple dissections and stroke, raising the question of whether these patients have a more severe vasculopathy. ${ }^{14,47}$ However, VWCE is not a specific indicator of dissection, because this finding can be seen in areas without other evidence of vessel pathology. ${ }^{3}$ Whether this represents an underlying vasculopathy that predisposes to dissection remains unclear.

\section{Blunt Cerebrovascular Injury}

Traumatic injury to the CA or VA is a major cause of stroke, especially in younger patients. As many as $2.4 \%$ of patients admitted with nonpenetrating trauma have radiographic evidence of BCVI; of these, 5\%-10\% experience a stroke. ${ }^{8,13,54,55,60}$ CTA is commonly used to screen at-risk trauma patients with excellent sensitivity. ${ }^{16,59}$ However, false-positive findings are common, particularly in lowgrade injuries, where the positive predictive value of CTA is only $30 \% .{ }^{19}$ Furthermore, as many as $42 \%$ of BCVIs identified with CTA are equivocal; of these $36 \%$ remain indeterminate on delayed repeat CTA. ${ }^{15}$ Given the risks of treatment with antiplatelet and antithrombotic agents, avoiding overdiagnosis is important. Although DSA remains the gold standard, this test is invasive and not without risk. VWI may help improve diagnostic accuracy in at-risk patients. A small pilot study found that VWI of- 

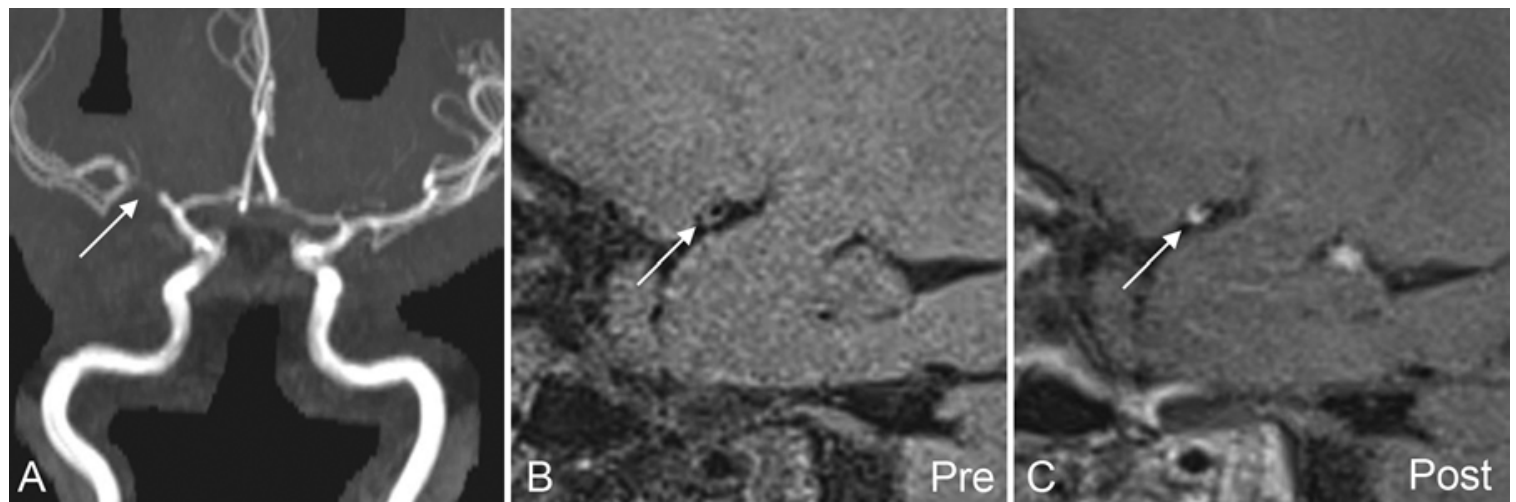

FIG. 5. Intracranial atherosclerosis. Coronal 3D maximum intensity projection MRA shows focal stenosis of the right MCA (A, arrow); however, underlying vasculopathy is unclear. Sagittal T1-weighted pre- and postcontrast VWI (B and C, respectively) shows eccentric wall thickening with enhancement and mild outward remodeling ( $\mathrm{B}$ and $\mathrm{C}$, arrows) compatible with intracranial atherosclerosis.

fered significantly better discrimination between equivocal findings and true BCVI, particularly in low-grade lesions ${ }^{65}$ Further research will be needed to better delineate the role of VWI in this population.

\section{Steno-Occlusive Cerebrovascular Vasculopathies}

\section{$\mathrm{CA}$ and Intracranial Atherosclerosis}

VWI provides a supplementary method for early diagnosis of intracranial and extracranial atherosclerosis and to target at-risk atherosclerotic lesions. Current treatment algorithms may be based on luminal stenosis, with symptoms and $70 \%$ stenosis of the extracranial carotid artery used as the threshold for endovascular treatment or carotid endarterectomy. ${ }^{4}$ However, VWI shows that high-risk atherosclerosis may not always be associated with significant stenosis. ${ }^{71}$ Angiography can underestimate the atherosclerosis burden, and does not detect nonstenotic lesions. VWI directly evaluates plaque, particularly the degree and pattern of vessel wall thickening. Indeed, increases in arterial VWCE correlate with age and atherosclerosis progression..$^{37}$ The typical atherosclerosis plaque is an eccentric, heterogeneous, contrast-enhancing, positive remodeling lesion (Fig. 5). Positive remodeling with an increase in vessel wall thickness as a compensatory dilatation, without initial changes in lumen diameter, can be missed by luminal imaging until later stages when stenosis occurs. ${ }^{32,71}$ Plaque rupture is often apparent at sites with only modest luminal stenosis but significant positive remodeling. Arteries with positive remodeling are more vulnerable to rupture than those with negative remodeling. ${ }^{12}$ With significant positive remodeling, chronic hypoperfusion may occur in the absence of significant stenosis. ${ }^{70}$

The layered pathological structure of atherosclerotic plaques resected via carotid endarterectomy correlates with VWI appearance. These specimens have juxtaluminal fibrous caps, lipid cores, and increased vasa vasorum ingrowth. Radiographically, these are demonstrated by a T2-hyperintense and T1-enhancing juxtaluminal layer, a T2-isointense, T1-hypointense nonenhancing middle layer, with a variable thickness enhancing outer layer, respectively. ${ }^{32}$ With increasing volume of a necrotic lipid core, there is an increased risk of rupture. In multifocal atherosclerotic disease, culprit lesions enhance more consistently than nonculprit lesions. The radial location of plaques along vessel walls can forecast stroke type. For example, plaques along the superior wall of the MCA are associated with lenticulostriate infarcts. ${ }^{37}$ Intraplaque hemorrhage has been shown to be one of the strongest markers for plaque vulnerability, and is represented by T1 shortening within the plaque.

Adding VWI to luminal imaging allows differentiation of atherosclerosis from other nonocclusive vasculopathies. In a recent study in which atherosclerosis, vasculitis, and reversible cerebral vasoconstriction syndrome (RCVS) lesions were evaluated, the addition of VWI to luminal imaging increased diagnostic accuracy from $43.5 \%$ to $96.3 \%$ compared with luminal imaging alone. ${ }^{40}$ Furthermore, $91 \%$ of atherosclerotic lesions were found to have an eccentric enhancing pattern, as opposed to the concentric enhancement found in vasculitis or RCVS ${ }^{40} \mathrm{VWI}$ has also shown reliability in interrater and interscan reproducibility for atherosclerotic plaque identification intracranially. ${ }^{41}$

\section{Moyamoya Vasculopathy}

Moyamoya disease (MMD) is an idiopathic bilateral narrowing of internal carotid artery (ICA) termini leading to chronic hypoperfusion and risk of hemorrhage. Moyamoya syndrome (MMS) is the unilateral or bilateral supraclinoid ICA stenosis secondary to vasculopathies including intracranial atherosclerosis, vasculitis, radiation, and neurofibromatosis. ${ }^{53}$ Pathological changes of MMD include fibrocellular thickening of the intima, including hyperproliferation of the vessel wall, active angiogenesis, and matrix accumulation. A small number of inflammatory cells are seen on histological studies. There is also irregular undulation of the internal elastic laminae, attenuation of the media, and a decrease in the outer diameter of the vessel. ${ }^{28}$

It is important to distinguish MMD from MMS, because MMD usually requires surgical revascularization. This is in contrast to MMS, where the underlying causes may be reversible with medication. Luminal imaging cannot reliably distinguish between MMD and MMS. On 

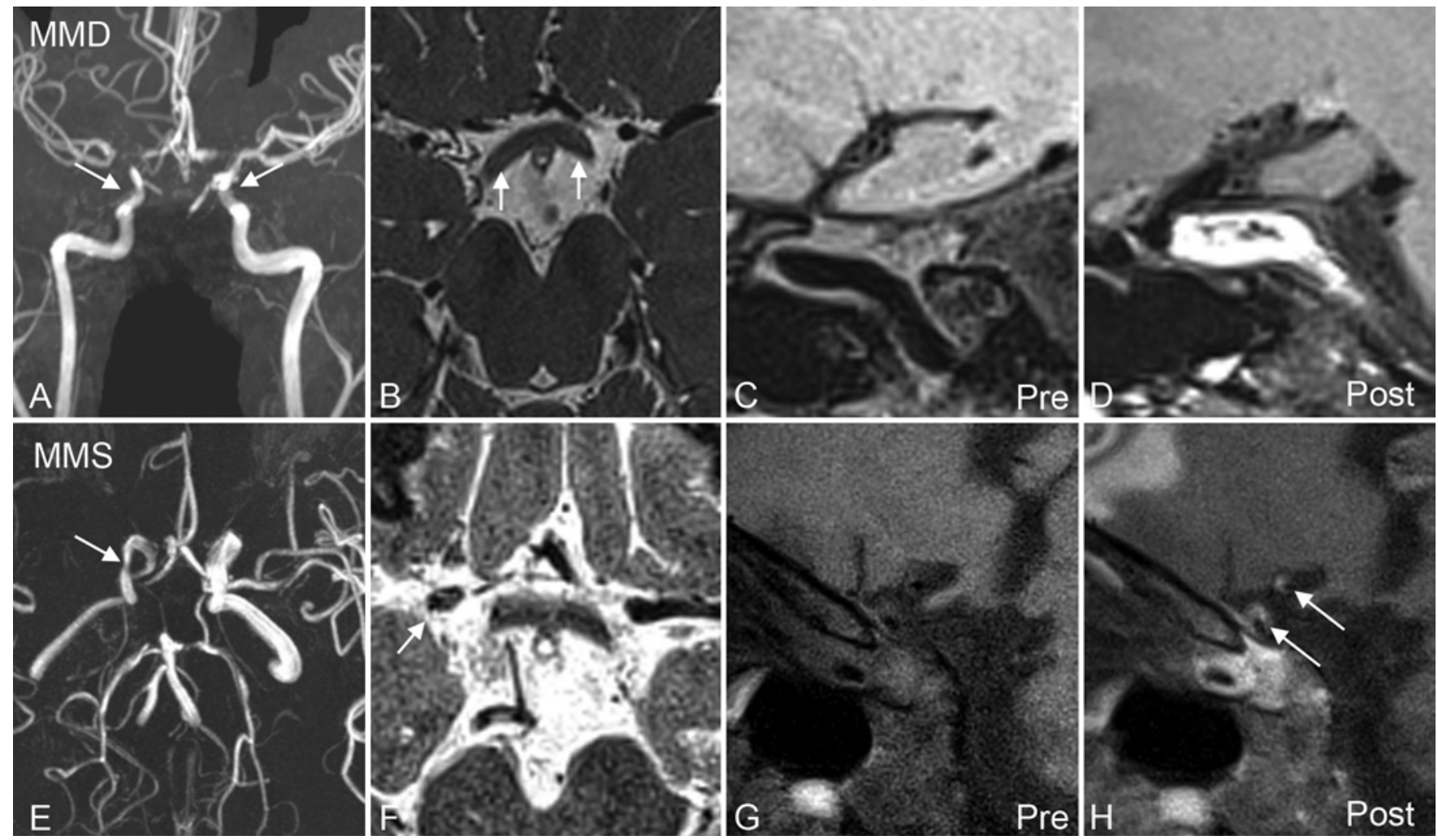

FIG. 6. VWI in MMD (A-D) and MMS (E-H). In MMD there is asymmetrical narrowing of the bilateral ICA terminus (arrows) and proximal anterior cerebral artery $(A C A)$ and $M C A(A)$. On T2-weighted VWI (B), there is no evidence of appreciable wall thickening. On T1-weighted pre- and postcontrast VWI (C and D, respectively), there is no evidence of outward remodeling, appreciable wall thickening, or wall signal abnormality. In MMS secondary to atherosclerosis, there is irregularity and asymmetrical cavernous and supraclinoid ICA stenosis ( $E$, arrow). On axial T2-weighted VWI $(F)$ there is wall thickening, with juxtaluminal T2 hyperintensity for the atherosclerotic fibrous cap and subjacent hypointensity for the lipid core (arrow). On T1-weighted pre- and postcontrast sagittal oblique VW images ( $\mathbf{G}$ and $\mathbf{H}$, respectively), there is outward remodeling plaque showing enhancement involving the $C A$ terminus and ACA (arrows).

VWI, MMS secondary to vasculopathy such as atherosclerosis will typically show focal eccentric lesion enhancement and outward remodeling (Fig. 6E-H). In contrast, segments affected by MMD tend to rarely enhance with no outward remodeling (Fig. 6A-D). When MMD segments do enhance, they have a mild concentric, homogeneous pattern. ${ }^{38}$ Another study directly comparing MMD and atherosclerotic vessel walls found bilateral distal ICA concentric enhancement and MCA wall shrinkage to be characteristic of MMD. ${ }^{49}$

VWCE in MMD may be an indicator of evolving intimal hyperplasia, neovascularization, and transient underlying inflammation correlating with disease instability and poor clinical outcomes. A recent cross-sectional study of 51 symptomatic patients with MMD found that patients with the highest degree of concentric VCWE had a higher incidence of acute ischemic stroke. The patient group with the highest enhancement had a $76 \%$ occurrence of acute ischemia, whereas the groups with minimal or no enhancement had 50\% and 48\%, respectively. ${ }^{69}$ However, the study did not distinguish between patients with MMD versus those with MMS, and included patients with unilateral and bilateral disease. Future prospective studies are needed to evaluate the clinical utility of VWI in differen- tiating MMD and MMS, and for predicting ischemic and hemorrhagic events, which could help stratify low- and high-risk patients and guide management decisions.

\section{CNS Vasculitis}

Conventional angiography may not be sensitive enough to detect the subtle changes in the distal cerebral vasculature affected by inflammatory vasculitis. VWCE in inflammatory vasculitis is theorized to be secondary to endothelial permeability and contrast leakage. ${ }^{32}$ VWI has shown the ability to detect small artery inflammatory vasculopathies. ${ }^{9}$ Contrast-enhanced MRI can detect enhancement in the parenchyma adjacent to the adventitia, representing inflammatory spillover, increasing the detection of affected segments, and may serve as an indicator of pathological microvascular involvement. ${ }^{1}$ On VWI, inflammation and edema within the vessel wall will show thickening and multifocal, homogeneous, and concentric enhancement. This is particularly useful for detecting a treatment response of presumed vasculitis after immunosuppressants. VWI may decrease the need for a high-risk brain tissue biopsy for CNS vasculitis, which has inherently low sensitivity. ${ }^{6}$ If a biopsy is indicated, VWI may help target a potentially high-yield lesion. ${ }^{37,38}$ 


\section{Reversible Cerebral Vasoconstriction Syndrome}

RCVS presents with sudden, severe headache and concomitant neurological deficits. Affected patients can develop infarction, SAH, edema, and intraparenchymal hemorrhage. In RCVS, VWI shows minimal smooth, concentric thickening with minimal diffuse or no enhancement. ${ }^{63}$ This appearance is consistent with the syndrome's pathophysiology, a noninflammatory transient vasospasm, and distinguishable from other causes of nonstenotic intracranial stenosis. A disproportionately greater increase in stenosis relative to the wall thickening is characteristic of vasospastic arteries. RCVS will show complete luminal resolution after approximately 3 months with conservative treatment. ${ }^{63}$ This contrasts with CNS vasculitis, which will usually demonstrate persistent VWCE and luminal narrowing despite treatment. . $^{32,37}$

\section{Conclusions}

High-resolution MR VWI complements traditional luminal and perfusion studies in the evaluation of cerebrovascular disease, and may allow earlier diagnosis and treatment, differentiate disease processes, and facilitate longitudinal evaluation. The clinical application for VWI is wide-ranging, answering important clinical questions and informing management decisions. Future prospective studies should be conducted to confirm the clinical benefit and accuracy associated with this imaging modality.

\section{Acknowledgments}

We appreciate the assistance of Sharon Durfy, PhD, with manuscript preparation.

\section{References}

1. Alexander MD, Yuan C, Rutman A, Tirschwell DL, Palagallo G, Gandhi D, et al: High-resolution intracranial vessel wall imaging: imaging beyond the lumen. J Neurol Neurosurg Psychiatry 87:589-597, 2016

2. Aoki S, Shirouzu I, Sasaki Y, Okubo T, Hayashi N, Machida T, et al: Enhancement of the intracranial arterial wall at MR imaging: relationship to cerebral atherosclerosis. Radiology 194:477-481, 1995

3. Arai D, Satow T, Komuro T, Kobayashi A, Nagata H, Miyamoto S: Evaluation of the arterial wall in vertebrobasilar artery dissection using high-resolution magnetic resonance vessel wall imaging. J Stroke Cerebrovasc Dis 25:14441450,2016

4. Barnett HJM, Taylor DW, Haynes RB, Sackett DL, Peerless SJ, Ferguson GG, et al: Beneficial effect of carotid endarterectomy in symptomatic patients with high-grade carotid stenosis. N Engl J Med 325:445-453, 1991

5. Bender MT, Wendt H, Monarch T, Beaty N, Lin LM, Huang $\mathrm{J}$, et al: Small aneurysms account for the majority and increasing percentage of aneurysmal subarachnoid hemorrhage: a 25 -year, single institution study. Neurosurgery 83:692-699, 2018

6. Berlit P, Kraemer M: Cerebral vasculitis in adults: what are the steps in order to establish the diagnosis? Red flags and pitfalls. Clin Exp Immunol 175:419-424, 2014

7. Bhogal P, Lansley J, Wong K, Udani SD, Uff C, Wadley J, et al: Vessel wall enhancement of a ruptured intra-nidal aneurysm in a brain arteriovenous malformation. Interv Neuroradiol 25:310-314, 2019

8. Bonow RH, Witt CE, Mosher BP, Mossa-Basha M, Vavilala
MS, Rivara FP, et al: Transcranial Doppler microemboli monitoring for stroke risk stratification in blunt cerebrovascular injury. Crit Care Med 45:e1011-e1017, 2017

9. Campi A, Benndorf G, Filippi M, Reganati P, Martinelli V, Terreni MR: Primary angiitis of the central nervous system: serial MRI of brain and spinal cord. Neuroradiology 43:599-607, 2001

10. Choi YJ, Jung SC, Lee DH: Vessel wall imaging of the intracranial and cervical carotid arteries. J Stroke 17:238-255, 2015

11. Chung JW, Kim BJ, Choi BS, Sohn CH, Bae HJ, Yoon BW, et al: High-resolution magnetic resonance imaging reveals hidden etiologies of symptomatic vertebral arterial lesions. J Stroke Cerebrovasc Dis 23:293-302, 2014

12. Cilla M, Peña E, Martínez MA, Kelly DJ: Comparison of the vulnerability risk for positive versus negative atheroma plaque morphology. J Biomech 46:1248-1254, 2013

13. Cogbill TH, Moore EE, Meissner M, Fischer RP, Hoyt DB, Morris JA, et al: The spectrum of blunt injury to the carotid artery: a multicenter perspective. J Trauma 37:473-479, 1994

14. Coppenrath E, Lenz O, Sommer N, Lummel N, Linn J, Treitl K, et al: Clinical significance of intraluminal contrast enhancement in patients with spontaneous cervical artery dissection: a black-blood MRI study. RoFo Fortschr Geb Rontgenstr Nuklearmed 189:624-631, 2017

15. Crawford JD, Allan KM, Patel KU, Hart KD, Schreiber MA, Azarbal AF, et al: The natural history of indeterminate blunt cerebrovascular injury. JAMA Surg 150:841-847, 2015

16. Eastman AL, Chason DP, Perez CL, McAnulty AL, Minei JP: Computed tomographic angiography for the diagnosis of blunt cervical vascular injury: is it ready for primetime? J Trauma 60:925-929, 2006

17. Edjlali M, Gentric JC, Régent-Rodriguez C, Trystram D, Hassen WB, Lion S, et al: Does aneurysmal wall enhancement on vessel wall MRI help to distinguish stable from unstable intracranial aneurysms? Stroke 45:3704-3706, 2014

18. Fan Z, Yang Q, Deng Z, Li Y, Bi X, Song S, et al: Wholebrain intracranial vessel wall imaging at 3 Tesla using cerebrospinal fluid-attenuated T1-weighted 3D turbo spin echo. Magn Reson Med 77:1142-1150, 2017

19. Grandhi R, Weiner GM, Agarwal N, Panczykowski DM, Ares WJ, Rodriguez JS, et al: Limitations of multidetector computed tomography angiography for the diagnosis of blunt cerebrovascular injury. J Neurosurg 128:1642-1647, 2018

20. Greving JP, Wermer MJH, Brown RD Jr, Morita A, Juvela $\mathrm{S}$, Yonekura M, et al: Development of the PHASES score for prediction of risk of rupture of intracranial aneurysms: a pooled analysis of six prospective cohort studies. Lancet Neurol 13:59-66, 2014

21. Habs M, Pfefferkorn T, Cyran CC, Grimm J, Rominger A, Hacker M, et al: Age determination of vessel wall hematoma in spontaneous cervical artery dissection: a multi-sequence 3T cardiovascular magnetic resonance study. J Cardiovasc Magn Reson 13:76, 2011

22. Harteveld AA, Denswil NP, Van Hecke W, Kuijf HJ, Vink A, Spliet WGM, et al: Ex vivo vessel wall thickness measurements of the human circle of Willis using 7T MRI. Atherosclerosis 273:106-114, 2018

23. Hartman JB, Watase H, Sun J, Hippe DS, Kim L, Levitt M, et al: Intracranial aneurysms at higher clinical risk for rupture demonstrate increased wall enhancement and thinning on multicontrast 3D vessel wall MRI. Br J Radiol 92:20180950, 2019

24. Hostettler IC, Alg VS, Shahi N, Jichi F, Bonner S, Walsh D, et al: Characteristics of unruptured compared to ruptured intracranial aneurysms: a multicenter case-control study. Neurosurgery 83:43-52, 2018

25. Hu P, Yang Q, Wang DD, Guan SC, Zhang HQ: Wall en- 
hancement on high-resolution magnetic resonance imaging may predict an unsteady state of an intracranial saccular aneurysm. Neuroradiology 58:979-985, 2016

26. Kalsoum E, Chabernaud Negrier A, Tuilier T, Benaïssa A, Blanc R, Gallas S, et al: Blood flow mimicking aneurysmal wall enhancement: a diagnostic pitfall of vessel wall MRI using the postcontrast 3D turbo spin-echo MR imaging sequence. AJNR Am J Neuroradiol 39:1065-1067, 2018

27. Krings T, Mandell DM, Kiehl TR, Geibprasert S, Tymianski $\mathrm{M}$, Alvarez $\mathrm{H}$, et al: Intracranial aneurysms: from vessel wall pathology to therapeutic approach. Nat Rev Neurol 7:547559,2011

28. Lehman VT, Brinjikji W, Mossa-Basha M, Lanzino G, Rabinstein AA, Kallmes DF, et al: Conventional and highresolution vessel wall MRI of intracranial aneurysms: current concepts and new horizons. J Neurosurg 128:969-981, 2018

29. Lindenholz A, van der Kolk AG, Zwanenburg JJM, Hendrikse J: The use and pitfalls of intracranial vessel wall imaging: how we do it. Radiology 286:12-28, 2018

30. Lv N, Karmonik C, Chen S, Wang X, Fang Y, Huang Q, et al: Relationship between aneurysm wall enhancement in vessel wall magnetic resonance imaging and rupture risk of unruptured intracranial aneurysms. Neurosurgery 84:E385-E391, 2019

31. Lv N, Tang H, Chen S, Wang X, Fang Y, Karmonik C, et al: Morphological parameters related to aneurysm wall enhancement in patients with multiple intracranial aneurysms. World Neurosurg 114:e338-e343, 2018

32. Mandell DM, Mossa-Basha M, Qiao Y, Hess CP, Hui F, Matouk $\mathrm{C}$, et al: Intracranial vessel wall MRI: principles and expert consensus recommendations of the American Society of Neuroradiology. AJNR Am J Neuroradiol 38:218-229, 2017

33. Matouk CC, Cord BJ, Yeung J, Malhotra A, Johnson MH, Minja FJ: High-resolution vessel wall magnetic resonance imaging in intracranial aneurysms and brain arteriovenous malformations. Top Magn Reson Imaging 25:49-55, 2016

34. Matouk CC, Mandell DM, Günel M, Bulsara KR, Malhotra A, Hebert R, et al: Vessel wall magnetic resonance imaging identifies the site of rupture in patients with multiple intracranial aneurysms: proof of principle. Neurosurgery 72:492-496, 2013

35. McGuinness BJ, Memon S, Hope JK: Prospective study of early MRI appearances following flow-diverting stent placement for intracranial aneurysms. AJNR Am J Neuroradiol 36:943-948, 2015

36. Mohr JP, Parides MK, Stapf C, Moquete E, Moy CS, Overbey JR, et al: Medical management with or without interventional therapy for unruptured brain arteriovenous malformations (ARUBA): a multicentre, non-blinded, randomised trial. Lancet 383:614-621, 2014

37. Mossa-Basha M, Alexander M, Gaddikeri S, Yuan C, Gandhi D: Vessel wall imaging for intracranial vascular disease evaluation. J Neurointerv Surg 8:1154-1159, 2016

38. Mossa-Basha M, de Havenon A, Becker KJ, Hallam DK, Levitt MR, Cohen WA, et al: Added value of vessel wall magnetic resonance imaging in the differentiation of moyamoya vasculopathies in a non-Asian cohort. Stroke 47:17821788,2016

39. Mossa-Basha M, Huynh TJ, Hippe DS, Fata P, Morton RP, Levitt MR: Vessel wall MRI characteristics of endovascularly treated aneurysms: association with angiographic vasospasm. J Neurosurg 131:859-867, 2019

40. Mossa-Basha M, Shibata DK, Hallam DK, de Havenon A, Hippe DS, Becker KJ, et al: added value of vessel wall magnetic resonance imaging for differentiation of nonocclusive intracranial vasculopathies. Stroke 48:3026-3033, 2017

41. Mossa-Basha M, Watase H, Sun J, Shibata DK, Hippe DS, Balu N, et al: Inter-rater and scan-rescan reproducibility of the detection of intracranial atherosclerosis on contrast-enhanced 3D vessel wall MRI. Br J Radiol 92:20180973, 2019

42. Nagahata S, Nagahata M, Obara M, Kondo R, Minagawa N, Sato S, et al: Wall enhancement of the intracranial aneurysms revealed by magnetic resonance vessel wall imaging using three-dimensional turbo spin-echo sequence with motionsensitized driven-equilibrium: a sign of ruptured aneurysm? Clin Neuroradiol 26:277-283, 2016

43. Natori T, Sasaki M, Miyoshi M, Ohba H, Oura MY, Narumi $\mathrm{S}$, et al: Detection of vessel wall lesions in spontaneous symptomatic vertebrobasilar artery dissection using T1weighted 3-dimensional imaging. J Stroke Cerebrovasc Dis 23:2419-2424, 2014

44. Omodaka S, Endo H, Fujimura M, Niizuma K, Sato K, Matsumoto Y, et al: High-grade cerebral arteriovenous malformation treated with targeted embolization of a ruptured site: wall enhancement of an intranidal aneurysm as a sign of ruptured site. Neurol Med Chir (Tokyo) 55:813-817, 2015

45. Omodaka S, Endo H, Niizuma K, Fujimura M, Endo T, Sato $\mathrm{K}$, et al: Circumferential wall enhancement on magnetic resonance imaging is useful to identify rupture site in patients with multiple cerebral aneurysms. Neurosurgery 82:638644, 2018

46. Pai SB, Varma RG, Kulkarni RN: Microsurgical anatomy of the middle cerebral artery. Neurol India 53:186-190, 2005

47. Pfefferkorn T, Saam T, Rominger A, Habs M, Gerdes LA, Schmidt C, et al: Vessel wall inflammation in spontaneous cervical artery dissection: a prospective, observational positron emission tomography, computed tomography, and magnetic resonance imaging study. Stroke 42:1563-1568, 2011

48. Portanova A, Hakakian N, Mikulis DJ, Virmani R, Abdalla WMA, Wasserman BA: Intracranial vasa vasorum: insights and implications for imaging. Radiology 267:667-679, 2013

49. Ryoo S, Cha J, Kim SJ, Choi JW, Ki CS, Kim KH, et al: High-resolution magnetic resonance wall imaging findings of Moyamoya disease. Stroke 45:2457-2460, 2014

50. Sakurai K, Miura T, Sagisaka T, Hattori M, Matsukawa $\mathrm{N}$, Mase M, et al: Evaluation of luminal and vessel wall abnormalities in subacute and other stages of intracranial vertebrobasilar artery dissections using the volume isotropic turbo-spin-echo acquisition (VISTA) sequence: a preliminary study. J Neuroradiol 40:19-28, 2013

51. Samaniego EA, Roa JA, Hasan D: Vessel wall imaging in intracranial aneurysms. J Neurointerv Surg 11:1105-1112, 2019

52. Schievink WI: Spontaneous dissection of the carotid and vertebral arteries. N Engl J Med 344:898-906, 2001

53. Scott RM, Smith ER: Moyamoya disease and moyamoya syndrome. N Engl J Med 360:1226-1237, 2009

54. Scott WW, Sharp S, Figueroa SA, Eastman AL, Hatchette CV, Madden CJ, et al: Clinical and radiographic outcomes following traumatic Grade 3 and 4 carotid artery injuries: a 10-year retrospective analysis from a Level 1 trauma center. The Parkland Carotid and Vertebral Artery Injury Survey. J Neurosurg 122:610-615, 2015

55. Scott WW, Sharp S, Figueroa SA, Eastman AL, Hatchette CV, Madden CJ, et al: Clinical and radiological outcomes following traumatic Grade 3 and 4 vertebral artery injuries: a 10-year retrospective analysis from a Level I trauma center. The Parkland Carotid and Vertebral Artery Injury Survey. J Neurosurg 122:1202-1207, 2015

56. Seo WK, Oh K, Suh SI, Seol HY: Clinical significance of wall changes after recanalization therapy in acute stroke: high-resolution vessel wall imaging. Stroke 48:1077-1080, 2017

57. Shimonaga K, Matsushige T, Ishii D, Sakamoto S, Hosogai M, Kawasumi T, et al: Clinicopathological insights from vessel wall imaging of unruptured intracranial aneurysms. Stroke 49:2516-2519, 2018 
58. Sikkema T, Uyttenboogaart M, Eshghi O, De Keyser J, Brouns R, van Dijk JMC, et al: Intracranial artery dissection. Eur J Neurol 21:820-826, 2014

59. Sliker CW: Blunt cerebrovascular injuries: imaging with multidetector CT angiography. Radiographics 28:1689-1710, 2008

60. Stein DM, Boswell S, Sliker CW, Lui FY, Scalea TM: Blunt cerebrovascular injuries: does treatment always matter? J Trauma 66:132-144, 2009

61. Swartz RH, Bhuta SS, Farb RI, Agid R, Willinsky RA, Terbrugge $\mathrm{KG}$, et al: Intracranial arterial wall imaging using high-resolution 3-tesla contrast-enhanced MRI. Neurology 72:627-634, 2009

62. Takano K, Yamashita S, Takemoto K, Inoue T, Kuwabara Y, Yoshimitsu K: MRI of intracranial vertebral artery dissection: evaluation of intramural haematoma using a black blood, variable-flip-angle 3D turbo spin-echo sequence. Neuroradiology 55:845-851, 2013

63. Tan HW, Chen X, Maingard J, Barras CD, Logan C, Thijs V, et al: Intracranial vessel wall imaging with magnetic resonance imaging: current techniques and applications. World Neurosurg 112:186-198, 2018

64. Texakalidis P, Hilditch CA, Lehman V, Lanzino G, Pereira VM, Brinjikji W: Vessel wall imaging of intracranial aneurysms: systematic review and meta-analysis. World Neurosurg 117:453-458.e1, 2018

65. Vranic JE, Huynh TJ, Fata P, Barber J, Bonow RH, Levitt MR, et al: The ability of magnetic resonance black blood vessel wall imaging to evaluate blunt cerebrovascular injury following acute trauma. J Neuroradiol [epub ahead of print], 2019

66. Wang GX, Li W, Lei S, Ge XD, Yin JB, Zhang D: Relationships between aneurysmal wall enhancement and conventional risk factors in patients with intracranial aneurysm: a high-resolution MRI study. J Neuroradiol 46:25-28, 2019

67. Wang GX, Wen L, Lei S, Ran Q, Yin JB, Gong ZL, et al: Wall enhancement ratio and partial wall enhancement on MRI associated with the rupture of intracranial aneurysms. J Neurointerv Surg 10:566-570, 2018

68. Wang J, Helle M, Zhou Z, Börnert P, Hatsukami TS, Yuan C: Joint blood and cerebrospinal fluid suppression for intracranial vessel wall MRI. Magn Reson Med 75:831-838, 2016
69. Wang M, Yang Y, Zhou F, Li M, Liu R, Guan M, et al: The contrast enhancement of intracranial arterial wall on highresolution MRI and its clinical relevance in patients with moyamoya vasculopathy. Sci Rep 7:44264, 2017

70. Xu WH, Li ML, Gao S, Ni J, Zhou LX, Yao M, et al: In vivo high-resolution MR imaging of symptomatic and asymptomatic middle cerebral artery atherosclerotic stenosis. Atherosclerosis 212:507-511, 2010

71. Xu WH, Li ML, Niu JW, Feng F, Jin ZY, Gao S: Intracranial artery atherosclerosis and lumen dilation in cerebral smallvessel diseases: a high-resolution MRI study. CNS Neurosci Ther 20:364-367, 2014

72. Yang H, Zhang X, Qin Q, Liu L, Wasserman BA, Qiao Y: Improved cerebrospinal fluid suppression for intracranial vessel wall MRI. J Magn Reson Imaging 44:665-672, 2016

73. Zolnourian A, Borg N, Akhigbe T, Macdonald J, Bulters $\mathrm{D}$ : Vessel wall imaging after subarachnoid hemorrhage in patients with multiple intracranial aneurysms: a cautionary case. World Neurosurg 127:414-417, 2019

\section{Disclosures}

Dr. Levitt has equity interest in eLoupes, Inc., Cerebrotech, and Corindus, and has unrestricted educational grants from Stryker, Medtronic, and Philips Volcano. Dr. Mossa-Basha has a research grant from Philips Healthcare. Dr. Kim is a shareholder of Spi Surgical, LLC, a surgical robotics company based in Seattle, WA.

\section{Author Contributions}

Conception and design: Levitt, Young, Mossa-Basha, Kim. Drafting the article: Young, Bonow, Barros. Critically revising the article: Levitt, Young, Mossa-Basha, Kim. Reviewed submitted version of manuscript: all authors. Approved the final version of the manuscript on behalf of all authors: Levitt.

\section{Correspondence}

Michael R. Levitt: c/o Neurosurgery Publications, University of Washington, Seattle, WA. respub@uw.edu. 